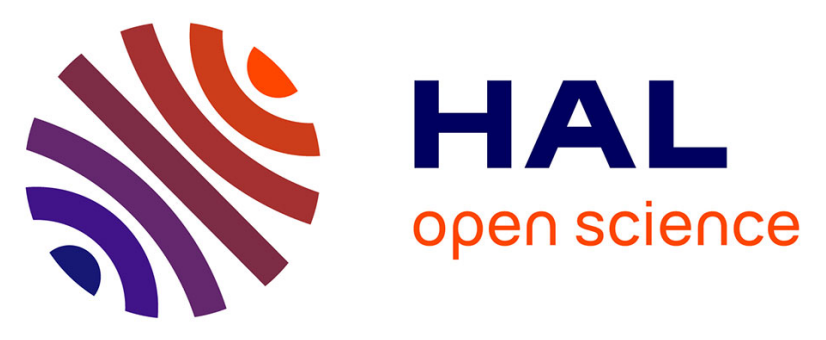

\title{
Electrical conductivity under shear flow of molten polyethylene filled with carbon nanotubes: Experimental and modeling
}

Anatole Collet, Anatoli Serghei, Olivier Lhost, Yves Trolez, Philippe Cassagnau, René Fulchiron

\section{To cite this version:}

Anatole Collet, Anatoli Serghei, Olivier Lhost, Yves Trolez, Philippe Cassagnau, et al.. Electrical conductivity under shear flow of molten polyethylene filled with carbon nanotubes: Experimental and modeling. Polymer Engineering and Science, 2021, 61 (4), pp.1129-1138. 10.1002/pen.25651. hal-03448412

\section{HAL Id: hal-03448412 \\ https://hal.science/hal-03448412}

Submitted on 25 Nov 2021

HAL is a multi-disciplinary open access archive for the deposit and dissemination of scientific research documents, whether they are published or not. The documents may come from teaching and research institutions in France or abroad, or from public or private research centers.
L'archive ouverte pluridisciplinaire HAL, est destinée au dépôt et à la diffusion de documents scientifiques de niveau recherche, publiés ou non, émanant des établissements d'enseignement et de recherche français ou étrangers, des laboratoires publics ou privés. 


\title{
Electrical conductivity under shear flow of molten Polyethylene filled with carbon nanotubes: experimental and Modeling
}

\author{
Anatole Collet ${ }^{1}$, Anatoli Serghei ${ }^{1}$, Olivier Lhost ${ }^{2},{\text { Yves } \text { Trolez }^{2} \text {, Philippe Cassagnau }}^{1}$, \\ René Fulchiron ${ }^{1 *}$ \\ ${ }^{1}$ Univ Lyon- Université Lyon 1, CNRS, Ingénierie des Matériaux Polymères (IMP-UMR \\ 5223), 15 Boulevard Latarjet, 69622 Villeurbanne Cedex (France) \\ ${ }^{2}$ Total Research and Technology Feluy, Zone Industrielle Feluy C, 7181 Feluy, Belgique
}

\begin{abstract}
This work aims to describe the conductivity evolution of polymer composites (polyethylene filled with carbon nanotubes) during a shearing deformation. Rheo-electric measurements were carried out to observe the shear-induced fillers network modification. Extended steady shear forces the conductivity to evolve asymptotically to a steady level attesting to an equilibrium between structuring and break up mechanisms in the melted polymer. Numerous experiments were conducted to cover a wide range of shear rate from $0.05 \mathrm{~s}^{-1}$ to $10 \mathrm{~s}^{-1}$ and for carbon nanotubes concentrations between 1.3 vol\% and 2.9 vol\%. A model is proposed to predict the conductivity evolution under shear deformation using a simple kinetic equation inserted in a percolation law. Structuring parameter was found to be solely dependent on the temperature whereas shear induced modification terms were found to be mostly driven by the shear rate and the fillers content.
\end{abstract}

Keywords:

Nanocomposites; melt; polyethylene (PE); shear; conducting polymers; modeling

\section{Introduction}

During last decades, carbon nanotubes (CNT) have proven their value as filler in polymer nanocomposites and find their place in numerous industrial applications [1-3]. This material can be used whether to improve mechanical properties [4-6] or to implement new features to a polymer matrix such as electrical conductivity [7-11]. Concerning the latter, the high aspect ratio of this material enables the improvement of a matrix with a theoretically low CNT content, less than one percent $[12,13]$.

In practice, the percolation threshold occurs at higher concentration [14, 15]. Nanotubes are characterized by a high aspect ratio with a contorted shape. They are generally aggregated and the structure of the network resulting from the aggregates interconnections can vary significantly with the polymer matrix, the processing method and nanotube type [16]. Electrical properties rely on a continuous network composed by contacts between small aggregates and individual CNTs [17-20]. A contact here is considered as an electrical contact, which means an electrical charge transfers between CNT by any mechanism (including tunnel effect) [21-23]. This complex grid can be modified during processing steps (Thermoforming, mould injection, extrusion...) by the applied mechanical deformation [20, 24-26]. For cost reasons but also in-use mechanical properties, a very low CNT content is desired but this 
leads to electrical properties very dependent on processing steps and turns the manufacturing of conductive polymer composites into an industrial challenge [27].

To develop the comprehension of physical mechanisms responsible for the CNT network modification in melt polymer, rheological analysis coupled with conductivity measurement is valuable. A convenient way to monitor the CNT network in a polymer matrix is to measure the electrical response. This specific measurement allows full characterization of the conductivity characterization during quiescent treatment as well as during shear deformation.

In 2007, Obzrut used a rheoelectric set up and managed to monitor the destruction of the conductive network for polypropylene/CNT composites under steady shear [28]. The shear induced conductor to insulator transition [29] as well as the dynamic percolation [30] have now been observed under various condition and for different matrices. These phenomena depict the ability of the filler's network to evolve in a polymer melt whether to decrease or increase the number of CNT-CNT connections in the sample.

To describe the competition between structuring and destruction mechanisms in shear flow, a first model was proposed by Skipa et al. [31]. The evolution of the agglomerates fraction contributing to the electrical conductivity was expressed by the following kinetic equation:

$$
\frac{d \varphi_{e f f}}{d t}=k_{0}\left(\varphi-\varphi_{e f f}\right)^{n}+k_{1}\left(\varphi-\varphi_{e f f}\right)-k_{2} \varphi_{e f f}
$$

Where $\varphi_{\text {eff }}$ is the effective volume fraction of conductive agglomerates, $\varphi$ the filler volume fraction of agglomerates at the equilibrium $(t \rightarrow \infty)$ and $k_{0}, k_{1}$ and $k_{2}$ the kinetic parameters relating to the process of quiescent agglomeration, shear-induced agglomeration and shear induced destruction, respectively. In Eq. (i), a simple first order kinetics $(n=1)$ was taken for the quiescent agglomeration phenomenon. Moreover, it was postulated that the last two kinetic parameters depend on the shear rate $\dot{\gamma}$ but also on the CNT species in the sample (large and small agglomerates and separated nanotubes). This equation introduced in a percolation law well describes the measured conductivity evolution of polycarbonate-CNT composites under shearing. However, in this model, each of these CNT species is governed by its specific kinetic law, so that many fitting parameters are introduced with difficult decoupling due to the rather imprecise definition of the boundaries between the three species [31].

Recently Marcourt et al. [32] developed a model describing the conductivity evolution of a CNT filled composite under extensional deformation. They assumed that CNTs, whether they are regrouped as agglomerates or just individual, take part in the sample's electrical conductivity as long as they are connected to the interconnected network [20]. In other words, more than counting CNTs that are connected to each other by contacts, the model was developed by considering an efficient part of the volume fraction of CNTs and a nonefficient part. The efficient part contains the CNTs incorporated in the percolation network (providing an electrical path from one surface of the material to the other). Conversely, 
aggregates that may possibly be connected to each other but without any contact with this percolation network are considered in the non-efficient part. It follows that the kinetic laws simply govern the exchanges between both parts.

Using this approach, a kinetic equation was proposed to describe the response to an elongational deformation:

$\frac{d \varphi_{\text {eff }}}{d t}=k_{\text {build }}\left(\varphi-\varphi_{\text {eff }}\right)-2 k_{\text {break }} \dot{\varepsilon}^{2} \varphi_{\text {eff }} t$

where $\varphi_{\text {eff }}$ is the filler volume fraction that belongs to the percolated network, $\varphi$ the total filler volume fraction, $k_{\text {build }}$ and $k_{\text {break }}$ are respectively the structuring and break-up kinetic constants. The first one has the dimension of the inverse of time, and the second one is adimensional. $\dot{\varepsilon}$ is the extensional rate. In the case of extensional stress, they observed that only the structuring mechanism was dependent on temperature following the same dependence as the matrix viscoelastic behavior. Thus, it was concluded that the structuring mechanism was due to the molecular mobility around CNTs. As regards the network breaking, it was shown to be solely relative to the deformation, following a negative exponential law of the squared deformation. In this case, it was concluded that the elongation contributes only to the network destruction systematically leading to a conductivity loss for moderate and high elongation rates [32]. This model allowed the prediction of the electrical conductivity under extensional deformation but also the conductivity recovery after cessation of the deformation.

For shear flow, the impact of the deformation on the conductive network is still unclear. Contrary to what has been observed for elongation, the effect of deformation is not only the total destruction of the conductor network and a part of the fillers fraction remains efficient. In this work, based on the conductivity monitoring during shear deformation, the mechanisms of the CNTs network modification are described. A simple model is proposed to predict the conductivity variation for HDPE/CNT melted composites under shear deformation.

\section{Experimental}

\subsection{Samples}

Polyethylene filled with carbon nanotubes composites were prepared by melt blending using the masterbatch dilution method in a dynamic mixer HAAKE ${ }^{\mathrm{TM}}$ Rheomix QC. Multiwalled carbon nanotubes (MWCNT), NC7000 from Nanocyl, were used. They are characterized by an average diameter of $9.5 \mathrm{~nm}$, a mean length of $1.5 \mu \mathrm{m}$ and a volume resistivity of $10^{-4} \Omega . \mathrm{cm}$. A masterbatch containing Carbon nanotubes was diluted in a polyethylene matrix by direct mixing at $50 \mathrm{rpm}$ for 15 minutes at $200^{\circ} \mathrm{C}$ to obtain desired filler's volume fraction. The used high-density polyethylene (HDPE), XRT70 from Total, has a melt flow index $\left(5 \mathrm{~kg}, 190^{\circ} \mathrm{C}\right)$ of $0.7 \mathrm{~g} / 10 \mathrm{~min}$. Then, the material was compression molded at $200^{\circ} \mathrm{C}$ as disks of $25 \mathrm{~mm}$ diameter and $2 \mathrm{~mm}$ thick. The compression cycle in the press was of around 15 minutes at $200^{\circ} \mathrm{C}$ before the cooling down to room temperature. 


\subsection{Rheo-electrical set up}

Electrical and viscoelastic measurements were carried out on an ARES G2 rheometer (TA Instruments). To enable the simultaneous characterization, ring-plate geometry electrodes were used and connected to a B2987A Electrometer (Keysight technologies). This set-up was already used by Moreira et al. [19] at ambient temperature. The conductivity is measured perpendicularly to the shear plane. It allows the electrical resistance measurement owing to a constant thickness of the sample while applying a quasi-homogeneous shear rate throughout the sheared material. The higher and smaller diameters of the cell are respectively 25 and $22 \mathrm{~mm}$, inducing only $14 \%$ of strain difference in the analyzed sample. A picture of the rheological configuration can be seen in Figure 1. As the lower ring/electrode is rotating, the electrical signal is collected using a connector whereas the stationary upper electrode is directly connected to the electrometer.

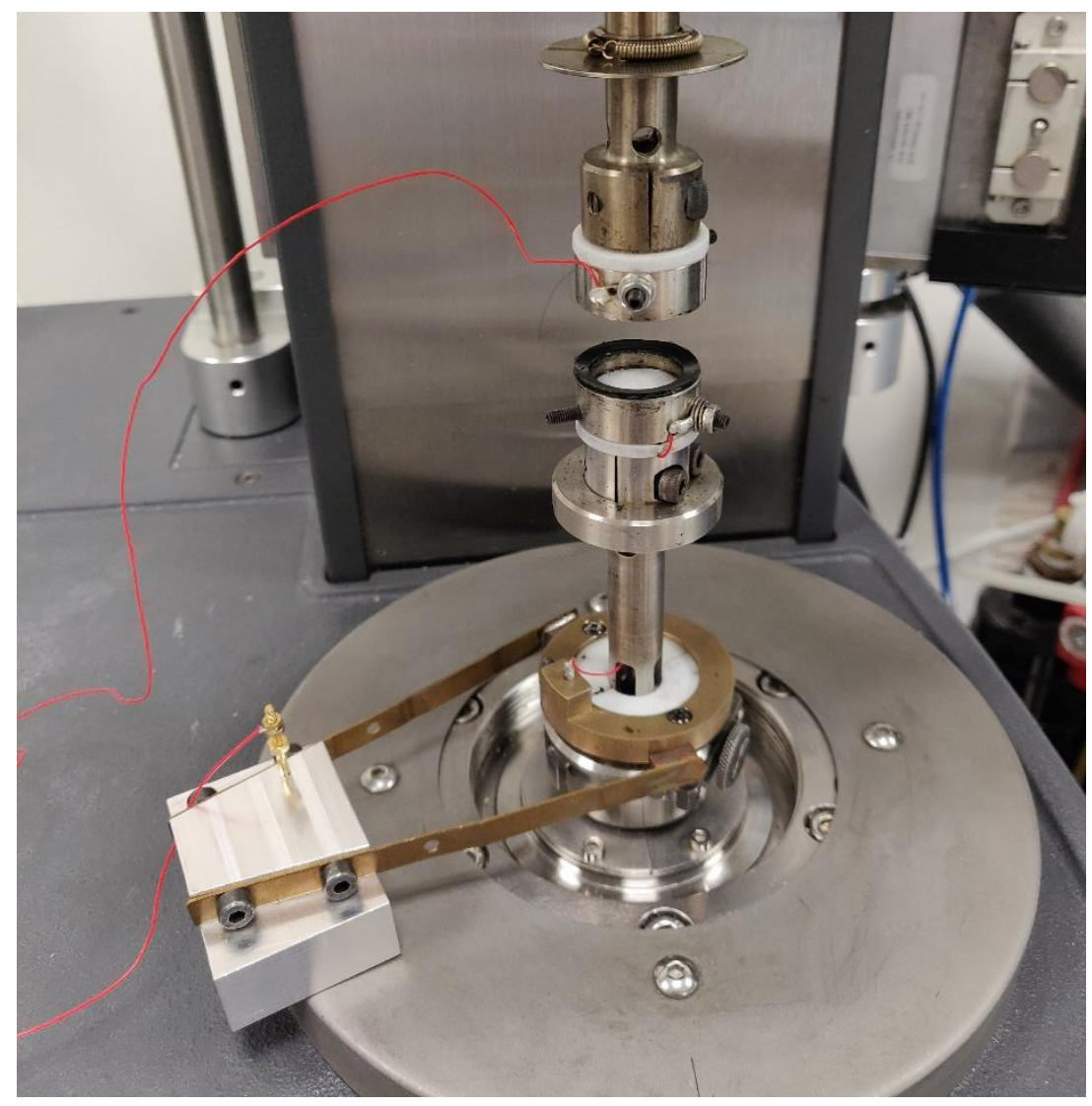

Figure 1: Picture of the rheological cell used for simultaneous conductivity measurement

A $1 \mathrm{~V}$ direct current (DC) was applied to the system and the resistance is measured every $0.1 \mathrm{~s}$. The volume conductivity $\sigma(\mathrm{S} / \mathrm{cm})$ is obtained using the geometry of the sample:

$\sigma=\frac{h}{S \cdot R}$

where $h$ is the thickness, $\mathrm{S}$ the surface of the ring (in our case equal to $2 \mathrm{~cm}^{2}$ ) and $R$ the measured resistance of the sample. The resistance of the short-circuited set-up was first measured, and the obtained value was around $1 \Omega$, which is negligible compared to the measured resistance of the studied materials. 
The samples were cut from pressed disks and placed between the ring cells. To ensure a good contact with both electrodes, temperature was set at $200^{\circ} \mathrm{C}$ to melt the polyethylene and the upper ring was brought in contact with the sample. The edge was removed to eliminate squeezed out material.

A quiescent treatment of around 15 minutes at $200^{\circ} \mathrm{C}$ was set until the sample conductivity regains a quasi-equilibrium state. Then a constant shear rate was applied to study the destruction and structuring process of the CNT network in the melt polymer.

As a preliminary analysis, the percolation law (Eq. (iv)) linking the measured conductivity to the effective CNT content was determined for annealed samples:

$\sigma=\sigma_{0}\left(\varphi_{\text {eff }}-\varphi_{c}\right)^{\beta}$

where $\varphi_{\text {eff }}$ is the effective CNTs volume fraction, defined as the filler concentration contributing to the conductivity of the sample. For these quiescent treatments, $\varphi_{\text {eff }}$ value was assumed to correspond to the total filler volume fraction $\varphi$ of the sample once the conductivity has reached a steady level. Experiments were carried out on specimens with a CNT content varying from 1.3 to 2.9 vol\%. Scilab software was used to fit the parameters by means of a non-linear least square regression subroutine. From these experiments fitting, the obtained value of the percolation threshold $\varphi_{c}$ is $1.15 \mathrm{vol} \%$, the exponent $\beta$ is 2 , and the prefactor $\sigma_{0}$ is $0.9 \mathrm{~S} . \mathrm{cm}^{-1}$.

\section{Description of the proposed model}

\section{Structuring induced by quiescent annealing}

The conductivity of a polymer-CNT composite is determined by the fraction of fillers that are interconnected but above all that are linked to the percolated network which offers an electrical pathway through the material. In molten polymer, annealing allows isolated CNTs to reconnect with the conductive network. In the case of samples that are not in the equilibrium (initial effective volume fraction, $\varphi_{\text {eff }}^{\text {initial }} \neq \varphi$ ), thermal diffusion of the polymer surrounding the CNTs enables the conductivity recovery [32].

In Eq. (iv), it is assumed that only the carbon nanotubes fraction that contributes to this network is accountable for the electrical conductivity. Isolated disentangled CNT and agglomerates detached from the network are inactive for the electrons transport.

Previous works presented models describing well the asymptotical behavior of conductivity in polymer-CNT composite during quiescent treatment. According to Marcourt et al. [32], the evolution of the effective fraction of CNTs in the sample is given by the following kinetic equation:

$\left.\frac{d \varphi_{\text {eff }}}{d t}\right|_{\text {structuring }}=k_{\text {build }}\left(\varphi-\varphi_{\text {eff }}\right)$

where $\varphi$ is the total filler fraction; $\varphi_{\text {eff }}$ is the filler fraction connected in the conductive network, necessarily limited to $\varphi$. For a given matrix the parameter $k_{\text {build }}$ is only dependent 
on the annealing temperature and is directly linked to the relaxation time of the polymer matrix.

The analytical solution of this equation allows a direct correspondence between effective concentration and annealing time:

$\varphi_{\text {eff }}=\varphi+\left(\varphi_{0}-\varphi\right) e^{-k_{\text {build }} \cdot t}$

where $\varphi_{0}$ corresponds to the initial effective filler fraction (which differs from $\varphi$ when the material is not at the equilibrium).

In this study the $k_{\text {build }}$ parameter was determined experimentally around the value of $1.3 \times$ $10^{-2} \mathrm{~s}^{-1}$ by fitting Eq. (vi) combined with Eq. (iv) on conductivity measured curves during the annealing of previously sheared samples. This value turned out to be suitable for all experiments carried out at $200^{\circ} \mathrm{C}$ with all the tested filler concentrations, independently of the deformation history. The impact of temperature on the structuring parameter has been verified by analyzing the conductivity recovery at 180 and $160^{\circ} \mathrm{C}$ of pre-sheared samples. The parameter $k_{\text {build }}$ was deduced to be $1 \times 10^{-2}$ and $7 \times 10^{-3} \mathrm{~s}^{-1}$ respectively. Figure 2 shows a comparison of the model and the experiments after various pre-shear for the three tested temperatures. These results are in accordance with the assumption that the molecular mobility inside the polymer has a prevailing role in the structuring mechanism. These parameter values will therefore be fixed in the following for modeling the results with the same matrix at these three temperatures of experiments.

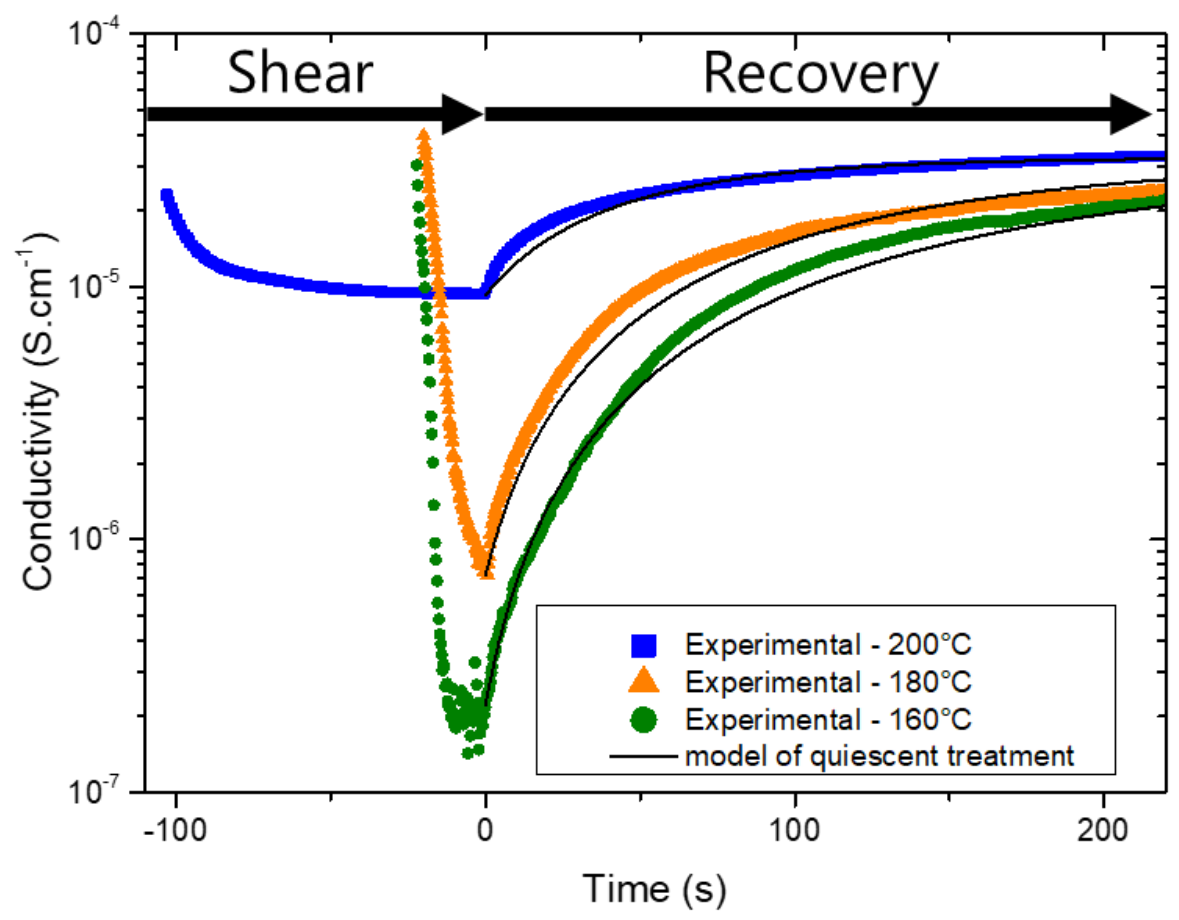

Figure 2: Conductivity evolution of a HDPE/CNT (1.75 vol\% MWCNT) under shear deformation (time <0 s) and during following quiescent treatment (time $\geq 0 \mathrm{~s}$ ) for various temperatures. Black lines are calculated curves using Eq. (vi). 


\section{Network modification under shear stress}

Deformation can alter the conductivity of a sample by modifying the conductive filler network structure, orientation, or dispersion [31]. For example, in elongation, the CNTs are strained apart from each other, destructing fillers connections hence triggering a conductor to insulator transition [20]. Conversely, in the case of shear deformation, both increase and decrease in conductivity can be achieved. During a shear experiment, the effective concentration evolves monotonically depending on the initial network structure and the applied deformation. Alig showed on insulating specimens that steady shear can induce a dynamic percolation up to a stationary conductivity value [31]. On the other side, a shear deformation can also trigger a reduction in the conductivity of a sample with an initially wellconnected CNT network [31]. In any case, the steady conductivity level reached during a shear deformation is found lower than in the quiescent melt. This stationary state is resulting from mechanisms of connection-disconnection of CNT contact under a shear deformation.

In this work, for experiments under shear leading to a steady conductivity, this steady level was always lower than this obtained for quiescent treatments (regardless of the shear rate, the temperature, or the CNT content). Moreover, for samples taken directly from the pressing operation i.e. which are out of equilibrium $\left(\varphi_{\text {eff }}^{\text {initial }}<\varphi\right)$, the recovery was slower when a shear was applied. For these reasons, rather than introducing a shear induced structuring parameter, the shear is considered here only as causing breakup. However, at long times, and contrary to what was obtained for elongation, a finite effective fraction $\left(\varphi_{\infty}\right)$ is achieved. In the material, this fraction can be considered as the result of continuous removals and collisions of CNT aggregates leading to a kind of equilibrium under shear. Thus, the impact of a shear flow on the effective filler fraction is considered according to the following kinetic equation:

$\left.\frac{d \varphi_{\text {eff }}}{d t}\right|_{\text {shearing }}=-k_{\text {break }}\left(\varphi_{\text {eff }}-\varphi_{\infty}\right)$

where $k_{\text {break }}$ is the breaking parameter with a dimension of an inverse time; $\varphi_{\infty}$ the equilibrium filler efficient fraction. Both parameters evolution with the shear rate will be discussed in the next section. Overall, the time derivative evolution of the effective CNT concentration can be simply expressed as the sum of both structuring and shearing equations:

$\frac{d \varphi_{\text {eff }}}{d t}=k_{\text {build }}\left(\varphi-\varphi_{\text {eff }}\right)-k_{\text {break }}\left(\varphi_{\text {eff }}-\varphi_{\infty}\right)$

This differential equation has the following analytical solution:

$\varphi_{\text {eff }}=\frac{k_{\text {build }} \cdot \varphi+k_{\text {break }} \varphi_{\infty}}{k_{\text {build }}+k_{\text {break }}}+\frac{\left(\varphi_{0}-\varphi_{\infty}\right) k_{\text {break }}+\left(\varphi_{0}-\varphi\right) k_{\text {build }}}{k_{\text {build }}+k_{\text {break }}} e^{-\left(k_{\text {build }}+k_{\text {break }}\right) \cdot t}$ 
From the coupling of this equation and the previous percolation equation (Eq. (iv)), the conductivity evolution as a function of time can be calculated and compared to the measured curves. The value of the initial effective filler fraction $\varphi_{0}$ was determined using the measured initial conductivity. The parameters $k_{\text {break }}$ and $\varphi_{\infty}$ were then fitted to experimental data with the use of a nonlinear least square regression algorithm (the parameter $k_{\text {build }}$ was already determined from quiescent measurements).

For $k_{\text {break }} \ll k_{\text {build }}$, the effective filler concentration follows the same evolution as a quiescent treatment, but for sufficient shearing forces, both structuring and destruction parameters will contribute to the network modification.

\section{Results and discussion}

4.1 fitting the evolution of conductivity during shear stress

The rheo-electrical set-up was configured to measure the sample resistance every $0.1 \mathrm{~s}$, allowing an accurate direct monitoring of the conductivity evolution during shear. To observe the destruction mechanism, the samples were first subjected to an annealing, so that the initial conductivity tended to the total filler concentration.

As a first illustration of the shear rate impact on the competition between structuring and destruction mechanisms, deformations up to five were applied to a HDPE/CNT (2 vol\% MWCNT) sample at different shear rates. Figure 3 shows the measured and calculated electrical conductivities as a function of time for start-up shearing experiments at different shear rates varying from $0.1 \mathrm{~s}^{-1}$ to $1 \mathrm{~s}^{-1}$. For these experiments, the shear was started when the sample has reached approximately the same conductivity $\left(9 \times 10^{-5} \mathrm{~S} / \mathrm{cm}\right)$ after annealing. The obtained conductivity decrease is explained by the destruction of CNT-CNT connections induced by the flow removing aggregates from the conductive network leading to the reduction of the effective filler fraction.

Figure 3 also shows that faster shear rates correspond to a lower steady conductivity level during shear. This result, in accordance with previous observation [31], shows the presence of destructive mechanisms during shear and their intensification with increasing $\dot{\gamma}$. The resulting conductivity loss is more abrupt, and the destruction mechanisms are stronger.

It can be mentioned that the calculated results well predict the limiting conductivity under shear when adjusting only the $k_{\text {break }}$ and $\varphi_{\infty}$ parameters. The fitted parameter values are summarized in Table 1 . These values clearly indicate that $k_{\text {break }}$ increases with the shear rate while $\varphi_{\infty}$ decreases in the range of experiments. The following section discusses the possible rationalization of these two parameters variations. 


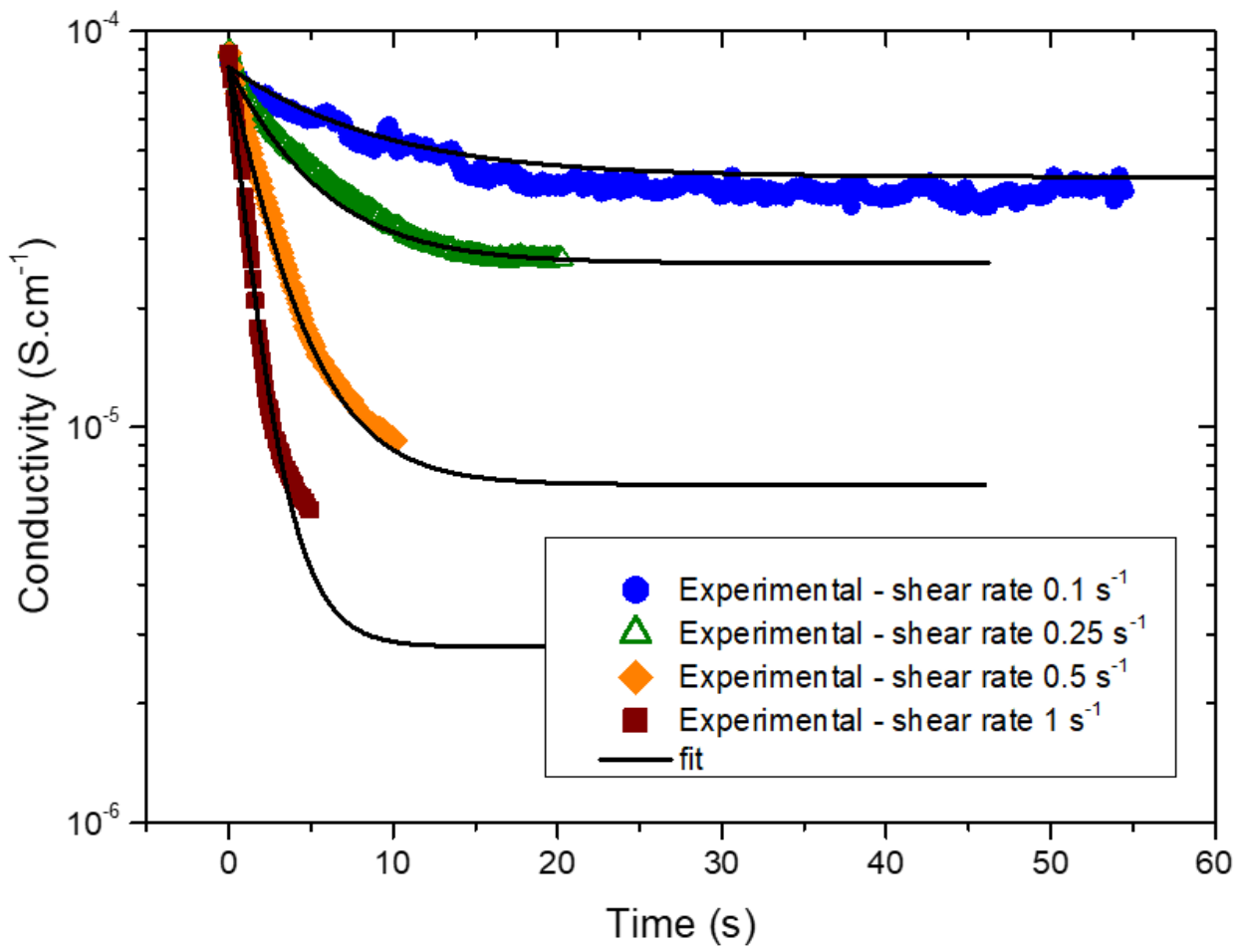

Figure 3: Conductivity variation during shear at $200^{\circ} \mathrm{C}$ as a function of time for HDPE/CNT (2.0 vol\%) at various shear rates for a total strain of 5. Symbols: experimental data; lines: calculated curves with Eq. (ix) and parameters shown in Table 1

Table 1: fitting parameters of different shear experiment of a HDPE-CNT (2.0 vol\% MWCNT) at $200^{\circ} \mathrm{C}$. The parameter $\boldsymbol{k}_{\text {build }}$ was fixed to $2.0 \times 10^{-2} \mathrm{~s}^{-1}$.

\begin{tabular}{|c|c|c|}
\hline Shear rate $\left(\mathrm{s}^{-1}\right)$ & $k_{\text {break }}\left(\mathrm{s}^{-1}\right)$ & $\varphi_{\infty}$ \\
\hline 0.1 & 0.10 & $1.8 \times 10^{-2}$ \\
\hline 0.25 & 0.19 & $1.7 \times 10^{-2}$ \\
\hline 0.5 & 0.29 & $1.4 \times 10^{-2}$ \\
\hline 1 & 0.55 & $1.3 \times 10^{-2}$ \\
\hline
\end{tabular}

\subsection{Shear rate dependence of the breaking parameter}

To understand the impact of shear rate on the breaking parameter $k_{\text {break }}$, various shear rates were applied and the conductivity response resulting from the shear was fitted with Eq. (ix). Shear rates from $0.05 \mathrm{~s}^{-1}$ to $10 \mathrm{~s}^{-1}$ were applied to samples with different CNT contents. In this respect, it can be mentioned that the choice of applied shear rates was related to the CNT contents because, for high contents (far above the percolation threshold), the applied shear rate must be high to give a significant conductivity decrease, whereas for CNT contents close to the threshold, a lower shear rate is sufficient. The resulting values of $k_{\text {break }}$ are shown in Figure 4 and clearly indicate a dependence on the shear rate. The error bars correspond to the standard deviation calculated for experiments that were reproduced up to seven times. This correlation indicates that the breaking parameter is controlled by the deformation rate and this dependence appears linear in the studied range (on a bi-logarithmic scale such as in Figure 4, this is shown by a slope equal to 1 ). Moreover, it can be seen in Figure 4 that the variations of $k_{\text {break }}$ with the shear rate merge for all the studied CNTs amounts. 


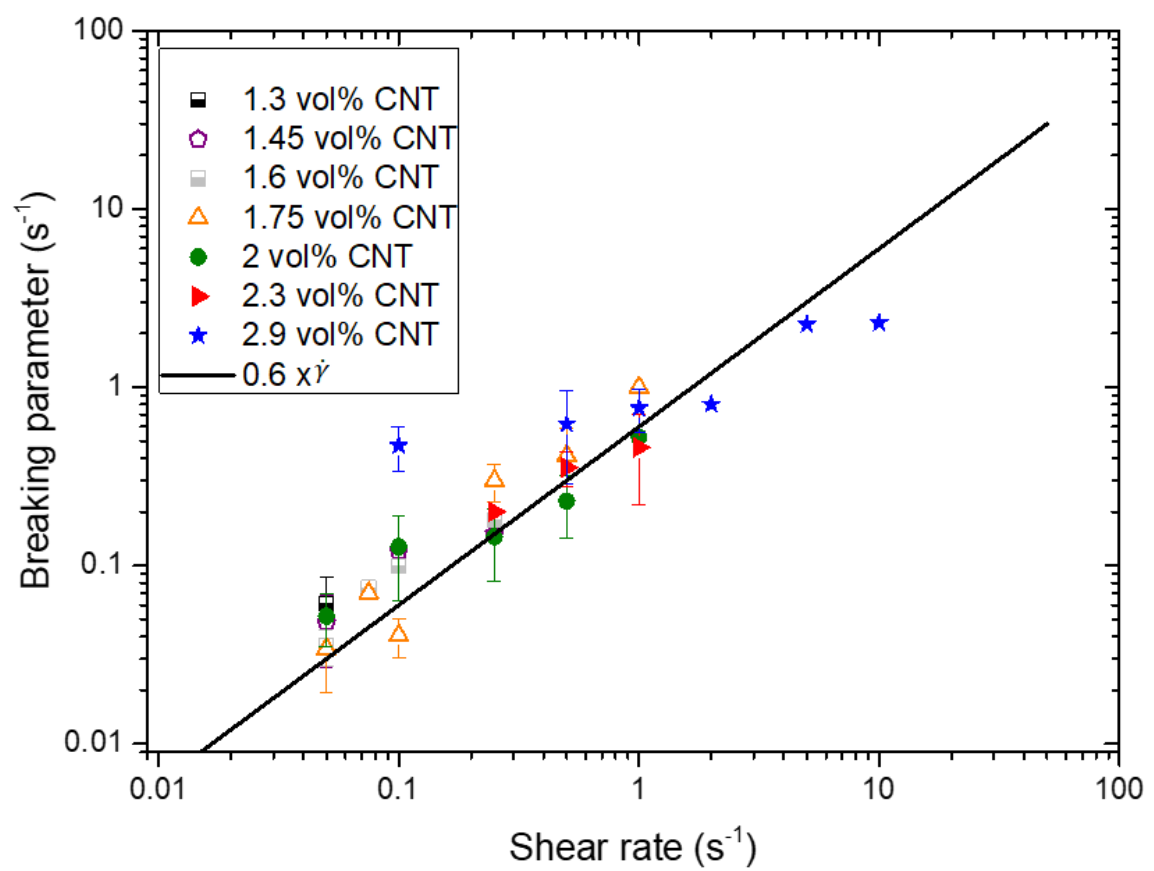

Figure 4: Adjusted break up parameter versus applied shear rate. Error bars are standard deviation obtained for repeated experiments.

The temperature of experiments was also varied to investigate its possible influence on the breaking parameter. As mentioned in a previous section, the temperature influences the building parameter $k_{\text {build }}$ and this parameter was fixed to the pre-evaluated values (according to the temperature). Then, the values of $k_{\text {break }}$ were adjusted. Figure 5 exhibits the calculated breaking parameter for HDPE/CNT (1.75 and 2.9 vol\% MWCNT) at 3 temperatures. In this graph, the adjusted $k_{\text {break }}$ evolves in the same way as a function of the shear rate, whether the experiments were carried out at 160,180 or $200^{\circ} \mathrm{C}$.

Nevertheless, it should be added that the conductivity final equilibrium value for a given shear rate is different from a temperature to another because the building process increases with the matrix mobility. Conversely, with increasing the shear rate, the conductive filler network undergoes faster partial destruction, and the equilibrium will occur at a lower conductivity due to a lower effective concentration. Therefore, from these results it can be concluded that the breaking mechanism is largely related to the shear rate, but it is independent of both temperature and filler amount. Therefore, for the following of this work, the breaking parameter is linked to the shear rate by a simple linear relation:

$$
k_{\text {break }}=\alpha \dot{\gamma}
$$

with $\alpha$ being an adimensional coefficient that was evaluated to 0.6 in this study. 


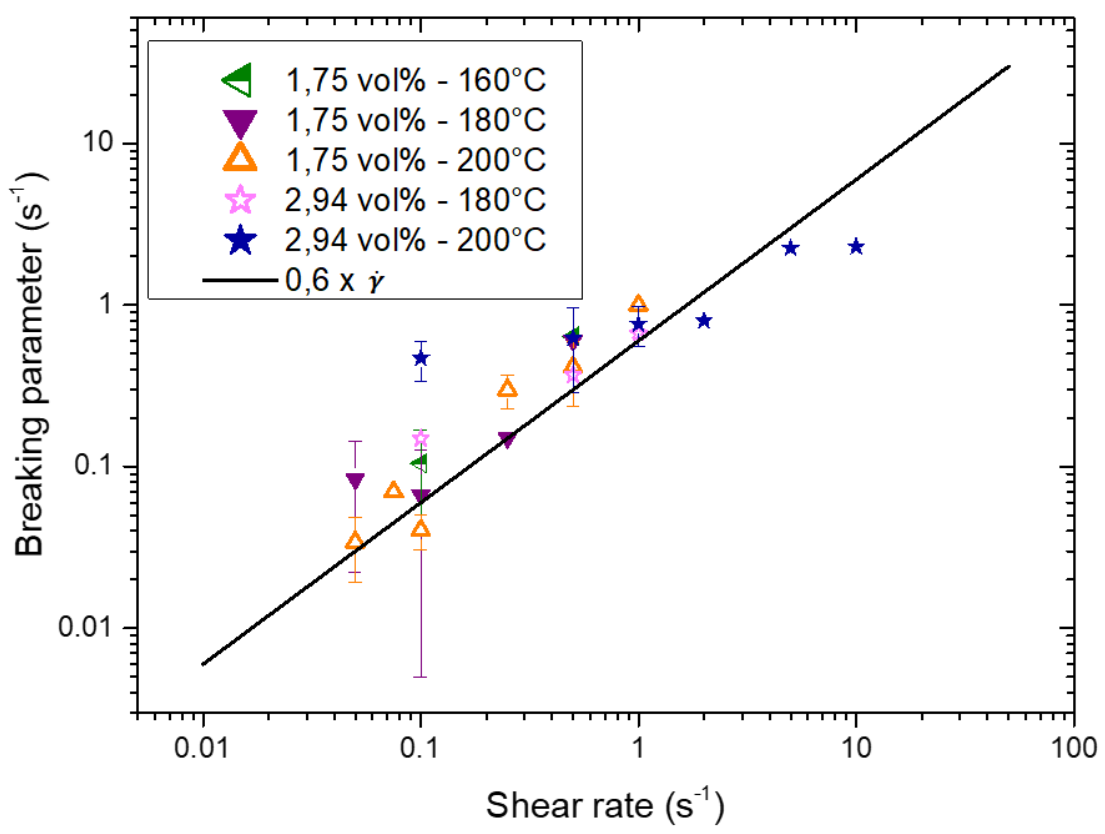

Figure 5: Adjusted break up parameter versus applied shear rate for experiments at different temperatures. Error bars are standard deviation obtained for repeated experiments.

It can be noticed that while $k_{\text {build }}$ parameter was found to be governed by the molecular mobility of the polymer matrix as already observed for polystyrene-CNT composites [32], the $k_{\text {break }}$ parameter seems highly linked to the nanotubes network. Here, only an unique polymer-filler system was studied (same CNT length, matrix, composite preparation...) but this breaking parameter will likely be modified for a different system.

\subsection{Variations of $\varphi_{\infty}$}

The value of both $k_{\text {build }}$ and $k_{\text {break }}$ parameters were thus determined and experimental data were fitted by adjusting again the $\varphi_{\infty}$ parameter. Suitable fittings were still obtained for the conductivity evolution during steady shear deformation. The obtained equilibrium filler fraction $\varphi_{\infty}$ is plotted versus the shear rate in Figure 6 for various CNT contents. The first obviousness from this plot is the clear dependence of this parameter on the sample total CNT content: for increasing filler fractions higher $\varphi_{\infty}$ values were determined.

The influence of the shear rate on $\varphi_{\infty}$ is mostly noticeable in Figure 6 for samples with a filler content of 2 vol\% (green filled circles). A decrease of the equilibrium efficient fraction from 1.8 vol\% to 1.15 vol\% was obtained over the entire applied shear rate range. The same trend is observed for other CNT contents, but with variable intensities. Taking the example of composites with a CNT concentration of 1.75 vol\%, $\varphi_{\infty}$ only shifts from 1.4 vol\% at $0.05 \mathrm{~s}^{-1}$ to 1.15 vol\% for highest shear rates. However, at high shear rates $\left(>1 \mathrm{~s}^{-1}\right)$, only a few experiments with the highest concentration (2.9 vol\%) were reliable in determining $\varphi_{\infty}$ so that a definitive interpretation of its variation for high shear rates would be impossible. 


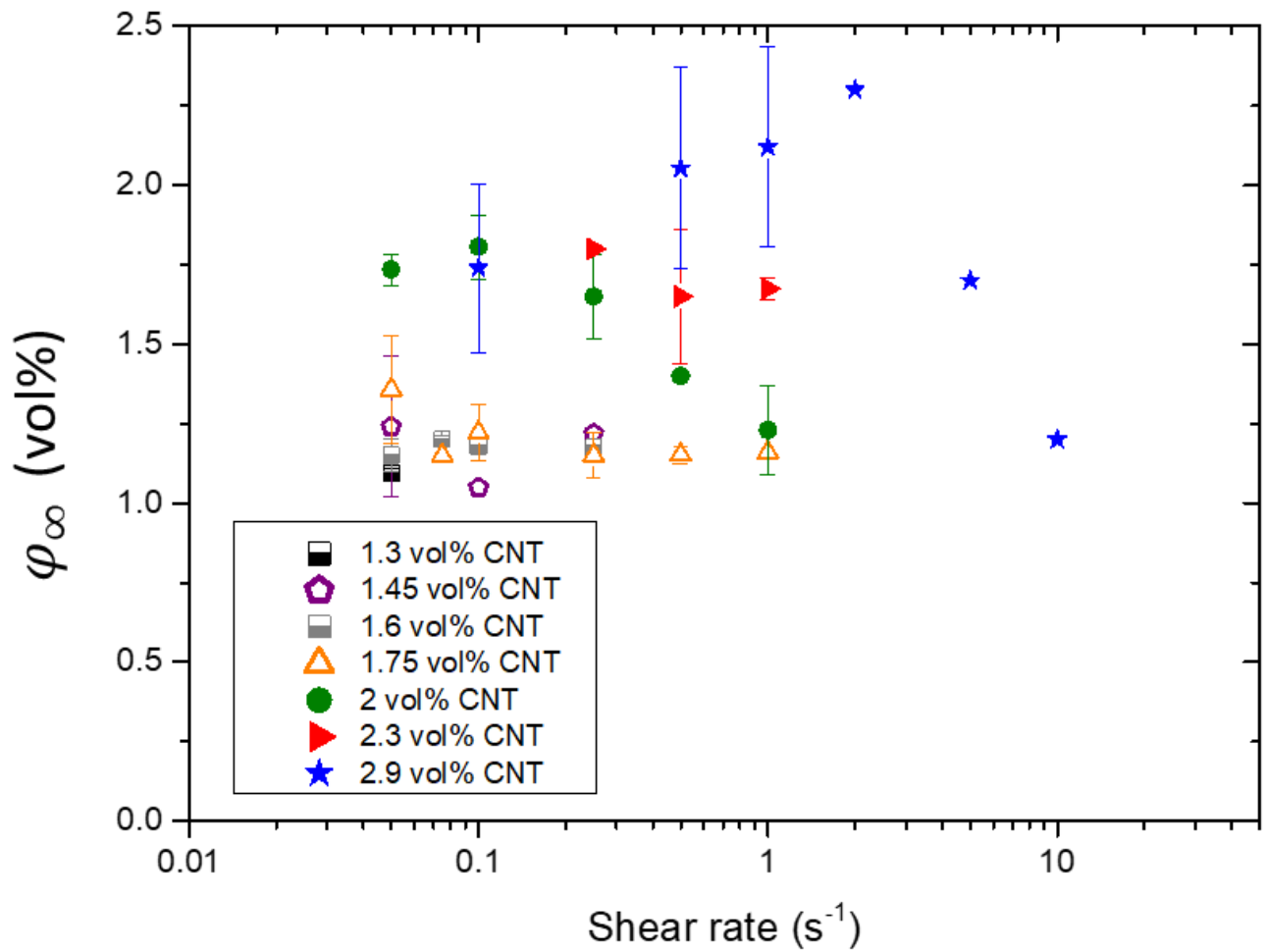

Figure 6: Equilibrium effective fraction versus shear rate for HDPE-CNT composites at various CNT content. The $\varphi_{\infty}$ parameter was adjusted from Eq(ix) with fixed break up and building parameters. Error bars are standard deviation obtained for repeated experiments.

In the model proposed by Skipa et al. [26] (Eq. (i)) a shear induced agglomeration term was used to describe the ability of CNT to agglomerate to the network. In this work, in Eq. (viii), the equilibrium effective filler fraction $\varphi_{\infty}$, is considered as a parameter that characterizes the shear induced destruction, balanced by a structuring, that occurs at long times of shear deformation. Under a shearing flow, the fillers are conveyed by the flowing matrix and this action mainly disconnects CNT-CNT contacts when the effective filler fraction is close to the total filler fraction $\left(\varphi_{e f f} \approx \varphi\right.$ ). If there is a non-negligible quantity of carbon nanotubes that are isolated from the conductive network $\left(\varphi_{e f f}<\varphi\right)$, the induced movement can help the reconnection to this network. The equilibrium effective fraction here testifies to the competition between shear induced creation and destruction of CNT contacts. The lower the $\varphi_{\infty}$ is, the more predominant is the break-up mechanism over the shear induced structuring.

The Temperature influence on the equilibrium efficient fraction was also investigated similarly to the previous section. The measured conductivity evolution with the shear rate at different temperatures has been fitted adjusting only $\varphi_{\infty}$. The determined values are plotted in Figure 7 showing that $\varphi_{\infty}$ appears independent of the temperature (but still dependent on the total CNT amount). In fact, this conclusion is consistent with the description of $\varphi_{\infty}$ which results from simultaneous removals and re-integrations of CNT aggregates in the conductive network due to the shear. If the re-integrations were due to the molecular mobility in the surrounding matrix, $\varphi_{\infty}$ would certainly be temperature dependent. 


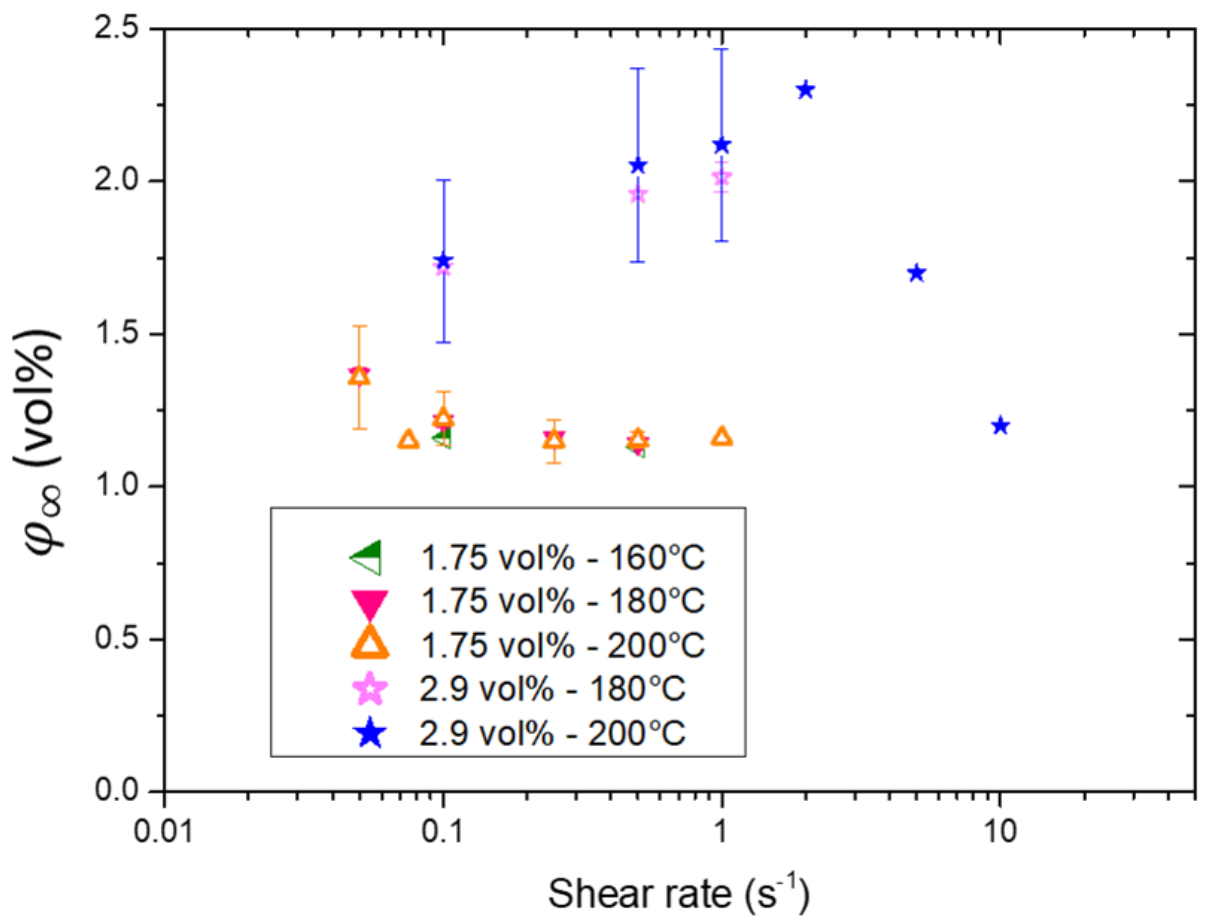

Figure 7: Equilibrium effective fraction versus applied shear rate for experiments at different temperatures. Error bars are standard deviation obtained for repeated experiments.

Thus, the equilibrium effective fraction was shown to be independent of the temperature, but it was observed in Figure 7 that this parameter is dependent on the total CNT amount $(\varphi)$. It remains difficult to determine a clear and strong variation of $\varphi_{\infty}$ with the shear rate. Nonetheless, when $\varphi_{\infty}$ presented in Figures 6 and 7 are normalized by the filler total fraction $\varphi$, a unique pattern, presented in Figure 8 , seems to emerge.

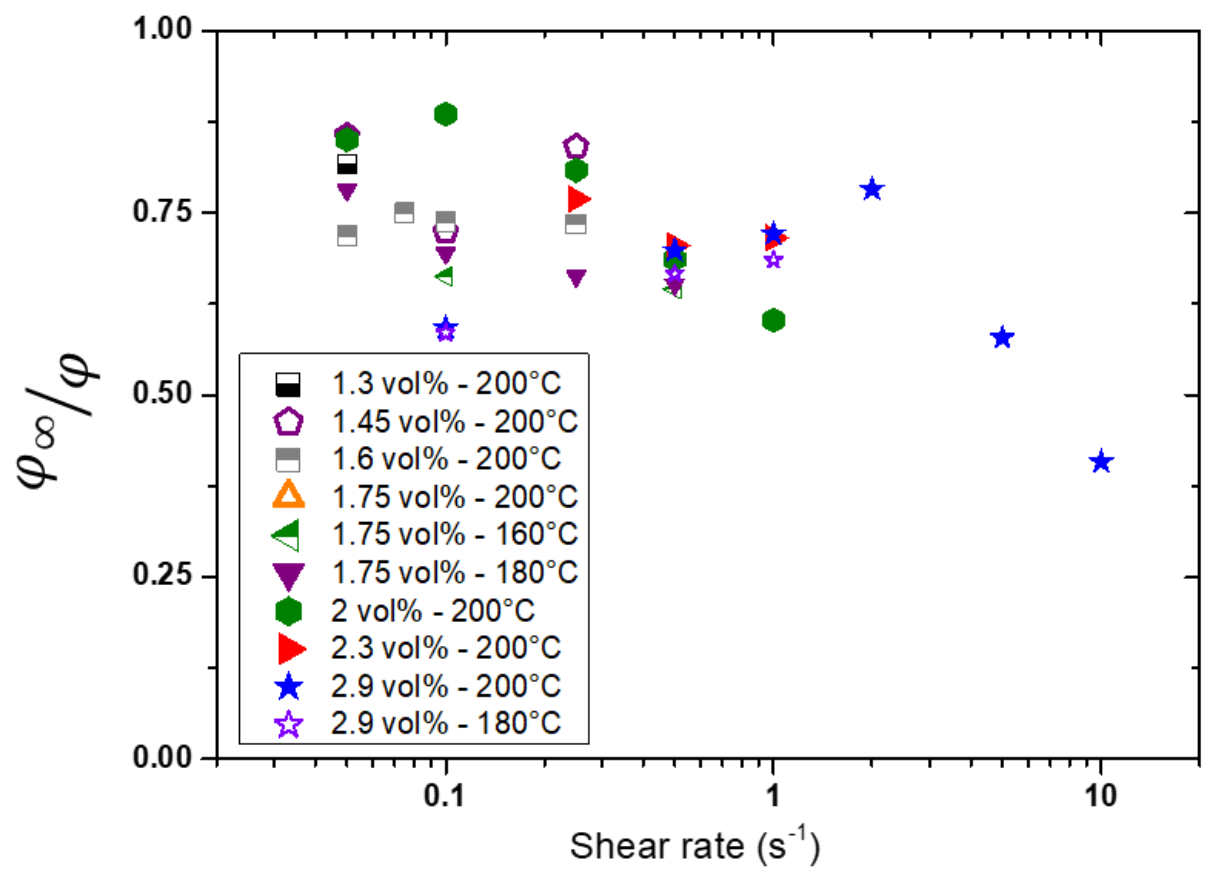

Figure 8: $\varphi_{\infty}$ parameter normalized by the total filler fraction $\varphi$ as a function of the shear rate for HDPE/CNT composites at various CNT contents. 
Nevertheless, at this stage, it would be pointless to attempt to establish a more precise direct quantitative relation between $\varphi_{\infty}$ and $\varphi$. Therefore, the individual $\varphi_{\infty}$ values obtained for given conditions of shear rate, temperature and CNT fraction will be used in the following section to further evaluate the proposed model.

\subsection{Modeling the electrical conductivity under shear flow}

As an illustrating example of the model validity, in Figure 9, the conductivity evolution measured at $200^{\circ} \mathrm{C}$ for a CNT fraction of 2 vol\% is compared to the data calculated with the model, and the parameters displayed in Table 2 (i.e. the breaking parameter $k_{\text {break }}$ is calculated with Eq. (x)). Even though small differences appear between measured and the calculated results, the model well predicts the shear induced conductivity evolution over a range from 0.1 to $1 \mathrm{~s}^{-1}$ for a composite with a 2 vol\% of CNT content.

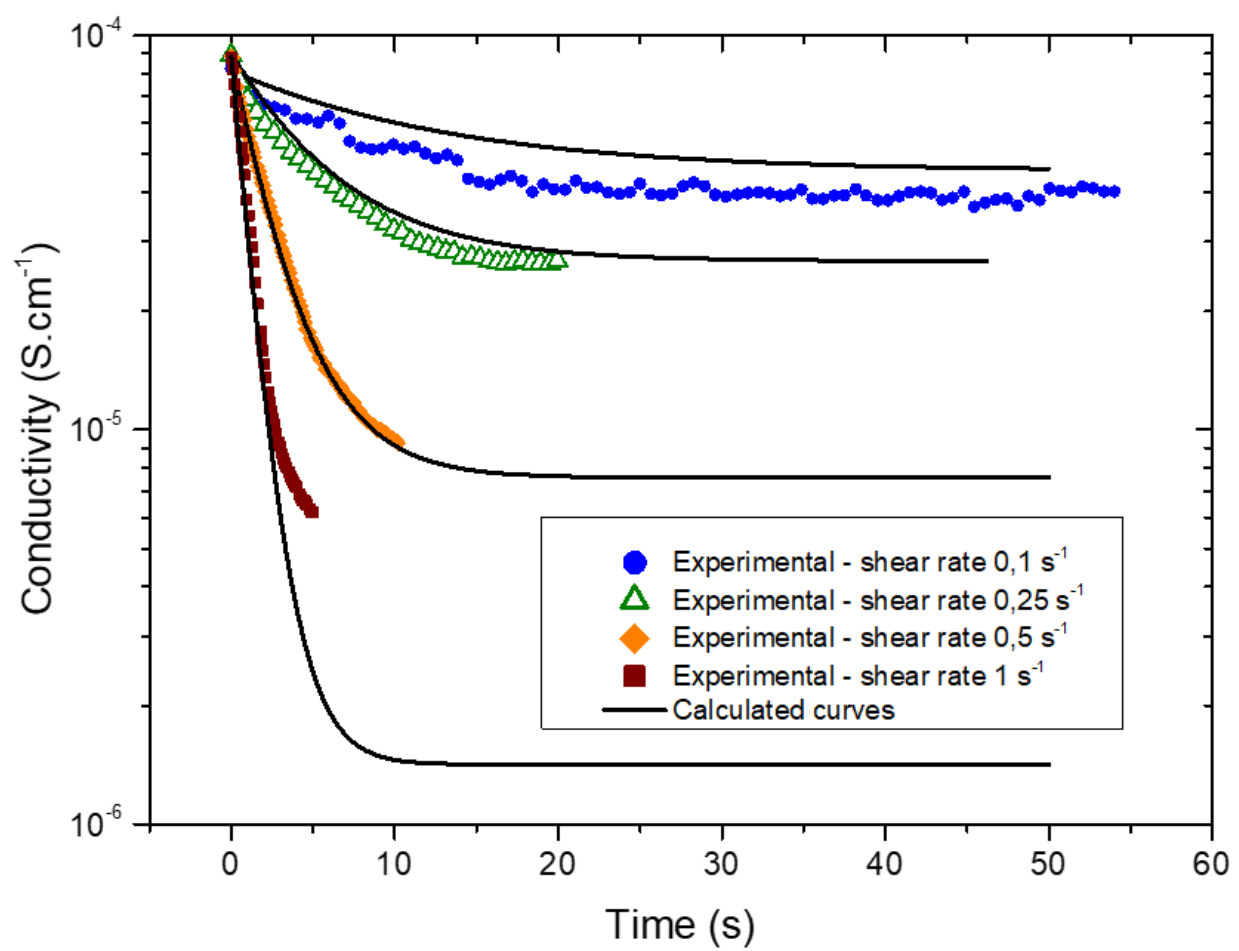

Figure 9: Conductivity as a function of time during shear deformation to 5 of HDPE/MWCNT (2 vol\%) at $200^{\circ} \mathrm{C}$ for different shear rates

Table 2 : Parameters value for the modeling of conductivity evolution during deformation of a HDPE/CNT (2 vol\% MWCNT) at $200^{\circ} \mathrm{C}$ for different shear rates. The parameter $\boldsymbol{k}_{\text {build }}$ was fixed to $2.0 \times 10^{-2} \mathrm{~s}^{-1}$

\begin{tabular}{|c|c|c|}
\hline Shear rate $\left(\mathrm{s}^{-1}\right)$ & $\boldsymbol{k}_{\text {break }}\left(\mathrm{s}^{-1}\right)$ & $\boldsymbol{\varphi}_{\infty}$ \\
\hline 0.1 & $6 \times 10^{-2}$ & $1.8 \times 10^{-2}$ \\
\hline 0.25 & 0.15 & $1.7 \times 10^{-2}$ \\
\hline 0.5 & 0.3 & $1.4 \times 10^{-2}$ \\
\hline 1 & 0.6 & $1.2 \times 10^{-2}$ \\
\hline
\end{tabular}

The reliability of the model was also tested for a more complex shear history. A HDPE/CNT (1.75 vol\% of MWCNT) was first annealed for 10 minutes. Then, a shear deformation was applied with successive lower and lower shear rates $\left(0.25 ; 0.1\right.$ and $0.05 \mathrm{~s}^{-1}$ ) for around 
50 seconds each and the electrical response was measured. The measured conductivity evolution is compared with the calculated data with the proposed model in Figure 10. The good accordance between both curves shows the ability of the model to well predict the conductivity evolution even for complex shearing histories.

During such an experiment, the initial effective concentrations ( $\varphi_{0}$ in Eq. (ix)) for the second and the third shear rate steps are different from this of the first step which is equal to the total fraction $\varphi$, considering the sample previously annealed. In fact, the initial effective fraction for one step is the ending effective fraction $\varphi_{\text {eff }}$ calculated for the previous step. Moreover, an increase of the electrical conductivity during both these steps was observed. The first shear step (shear rates of $0.25 \mathrm{~s}^{-1}$ for $46 \mathrm{~s}$ ) mainly causes CNT-CNT connections break-up, until the conductivity reaches a steady level corresponding to the equilibrium between structuring and destruction mechanisms. When the shear rate is then reduced (to $0.1 \mathrm{~s}^{-1}$ for $42 \mathrm{~s}$ and then to $0.05 \mathrm{~s}^{-1}$ for $58 \mathrm{~s}$ ), the conductivity evolves monotonically toward higher values. The breaking mechanism is reduced, and the network state is stabilized at a higher effective fraction. Consequently, the conductivity asymptotically increases. Once the shear is stopped, the breaking parameter equals to zero and the conductivity follows a quiescent recovery. Therefore, it is shown that, depending on the initial effective filler fraction an applied shear rate can either induce an increase or a decrease of the conductivity as it was already pointed out by Skipa et al. [31].

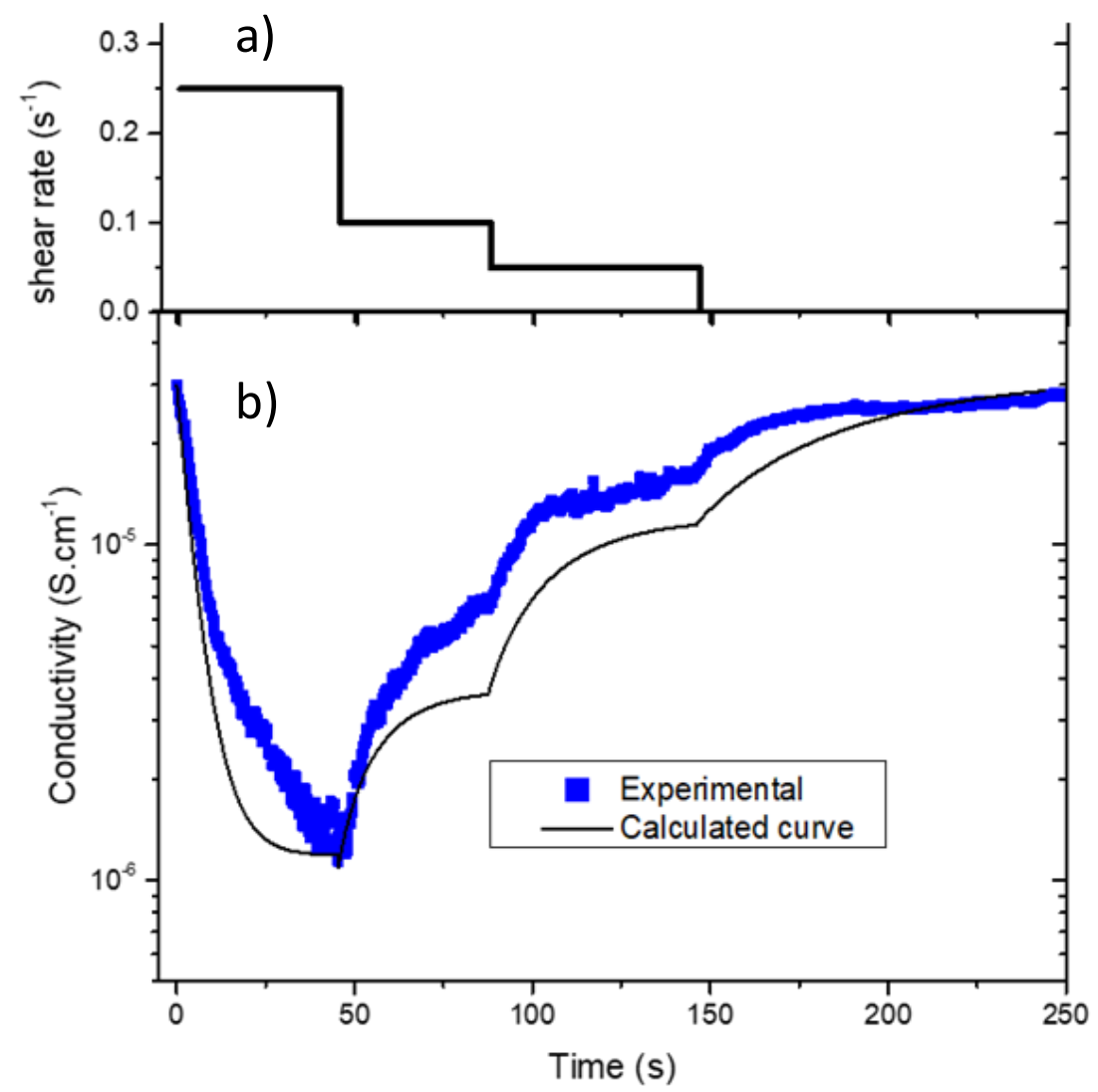

Figure 10: (a) Shear rate as a function of time. (b) Conductivity as a function of time of HDPE/MWCNT (1.75 vol\%) during deformation at successive lower and lower shear rates followed by a quiescent recovery at $200^{\circ} \mathrm{C}$. Symbols are experimental data and solid lines are model. 


\section{Conclusion}

The monitoring of the electrical conductivity was used in this work to study the building and breaking mechanisms of the carbon nanotubes network in a molten polymer under shear flow. The observations led to the proposition of a model describing the conductivity variation under shear flow for composites of polyethylene filled with carbon nanotubes. This model has been tested for materials with CNT contents nearby and above the percolation threshold (1.15 vol\% here) for a large range of shear rates and different temperatures. First, the structuring parameter, responsible for the conductivity enhancement under a quiescent annealing treatment, was determined. Then, the impact of the shear strain on the effective filler fraction for the HDPE/CNT composite was identified. It was observed that the breaking parameter was linearly dependent solely on the shear rate. An equilibrium effective fraction was introduced to report on the ability of the conductivity to stabilize at a certain level during the shear deformation. This factor was found to be related to the material total filler fraction and, to a lesser extent, to the applied shear rate. However, quantitative explanations of these tendencies are not entirely clear yet.

Finally, the proposed model gives the possibility to predict the conductivity evolution during shear deformation over a wide range of shear rate and filler concentrations regardless the initial state of the sample (possibly out of equilibrium). Considering that the parameters may be linked, for instance, to the quality of the dispersion as well as the type of CNT, the model might be adapted to other polymer-CNT composites.

\section{Acknowledgements}

This work was funded by the French National Association for Research and Technology (ANRT) and the company Total (CIFRE convention N²017/1593)

\section{References}

1 R. H. Baughman, A. A. Zakhidov and W. A. de Heer, "Carbon Nanotubes--the Route Toward Applications." Science, vol. 297, no. 5582, p. 787-792, 2002.

2 G. Mittal, V. Dhand, K. Y. Rhee, S. J. Park and W. R. Lee, "A review on carbon nanotubes and graphene as fillers in reinforced polymer nanocomposites." Journal of Industrial and Engineering Chemistry, vol. 21, p. 11-25, 2015

3 R. F. Gibson, "A review of recent research on mechanics of multifunctional composite materials and structures." Composite structures, vol. 92, no. 12, p. 2793-2810, 2010.

4 J. N. Coleman, U. Khan, W. J. Blau and Y. K. Gun'ko, "Small but strong: a review of the mechanical properties of carbon nanotube-polymer composites." Carbon, vol. 44, no. 9, p. 1624-1652, 2006. 
5 S. Mazinani, A. Ajji, C. Dubois, "Structure and Properties of Melt-Spun PET/MWCNT Nanocomposite Fibers." Polymer Engineering and Science, vol. 50, no. 10, p. 1956-1968, 2010.

6 J. Y. Kim, S. I. Han, S. H. Kim, "Crystallization behaviors and mechanical properties of poly(ethylene 2,6-naphthalate)/multiwall carbon nanotube nanocomposites." Polymer Engineering and Science, vol. 47, no. 11, p. 1715-1723, 2007.

7 H. Pang, L. Xu, D. X. Yan and Z. M. Li, "Conductive polymer composites with segregated structures." Progress in Polymer Science, vol. 39, no. 11, p. 1908-1933, 2014.

8 A. S. Zeraati, A. M. Anjaneyalu, S. P. Pawar, A. Abouelmagd, U. Sundararaj, "Effect of secondary filler properties and geometry on the electrical, dielectric, and electromagnetic interference shielding properties of carbonnanotubes / polyvinylidene fluoride nanocomposites." Polymer Engineering and Science, DOI:10.1002/pen.25591, Early Access Date: Dec. 2020

9 M. X. Lu, P. V. Gulgunje, P. J. Arias-Monje, J. Luo, J. Ramachandran, Y. Sahoo, S. Agarwal, S Kumar, "Structure, properties, and applications of polyacrylonitrile/carbon nanotube (CNT) fibers at low CNT loading." Polymer Engineering and Science, vol. 60, no. 9, p. 2143-2151, 2020.

10 M. R. Nobile, O. Valentino, M. Morcom, G. P. Simon, G. Landi, H. C. Neitzert, "The effect of the nanotube oxidation on the rheological and electrical properties of CNT/HDPE nanocomposites." Polymer Engineering and Science, vol. 57, no. 7, p. 665-673, 2017.

11 S. T. Zhou, A. N. Hrymak, M. R. Kamal, "Electrical and morphological properties of microinjection molded polystyrene/multiwalled carbon nanotubes nanocomposites." Polymer Engineering and Science, vol. 56, no. 10, p. 1182-1190, 2016.

12 E. J. Garboczi, K. A. Snyder, J. F. Douglas and M. F. Thorpe, "Geometrical percolation threshold of overlapping ellipsoids." Physical Review E, vol. 52, no. 1, p. 819-828, 1995

13 I. Balberg, N. Binenbaum and N. Wagner, "Percolation Thresholds in the Three-Dimensional Sticks System." Physical Review letters, vol. 52, no. 17, p. 1465, 1984.

14 J. Chen, B. Liu, X. Gao and D. Xu, "A review of the interfacial characteristics of polymer nanocomposites containing carbon nanotubes." RSC advances, vol. 8, no. 49, p. 28048-28085, 2018.

15 W. Bauhofer and J. Z. Kovacs, "A review and analysis of electrical percolation in carbon nanotube polymer composites." Composites Science and Technology, vol. 69, no. 10, p. 1486-1498, 2009.

16 Z. Spitalsky, D. Tasis, K. Papagelis and C. Galiotis, "Carbon nanotube-polymer composites: Chemistry, processing, mechanical and electrical properties." Progress in Polymer Science, vol. 35, no. 3, p. 357-401, 2020.

17 I. Alig, P. Pötschke, D. Lellinger, T. Skipa, S. Pegel, G. R. Kasaliwal and T. Villmow, "Establishment, morphology and properties of carbon nanotube networks in polymer melts." Polymer, vol. 53, no. 1, p. 4-28, 2012.

18 S. Pegel, P. Pötschke, G. Petzold, I. Alig, S. M. Dudkin and D. Lellinger, "Dispersion, agglomeration, and network formation of multiwalled carbon nanotubes in polycarbonate melts." Polymer, vol. 49, no. 4, p. 974-984, 2008.

19 L. Moreira, R. Fulchiron, G. Seytre, P. Dubois and P. Cassagnau, "Aggregation of Carbon Nanotubes in Semidilute Suspension." Macromolecules, vol. 43, no. 3, p. 1467-1472, 2010.

20 M. Marcourt, P. Cassagnau, R. Fulchiron, D. Rousseaux, O. Lhost and S. Karam, "An original combined method for electrical conductivity measurement of polymer composites under extensional deformation." Journal of Rheology, vol. 61, no. 5, p. 845-857, 2017. 
21 T. Takeda, Y. Shindo, Y. Kuronuma and F. Narita, "Modeling and characterization of the electrical conductivity of carbon nanotube-based polymer composites." Polymer, vol. 52, no. 17, p. 38523856, 2011.

22 C. Feng and L. Jiang, "Micromechanics modeling of the electrical conductivity of carbon nanotube (CNT)-polymer nanocomposites." Composites Part A: Applied Science and Manufacturing, vol. 47, p. 143-149, 2013.

23 Y. Zare and K. Y. Rhee, "A power model to predict the electrical conductivity of CNT reinforced nanocomposites by considering interphase, networks and tunneling condition." Composites Part B: Engineering, vol. 155, p. 11-18, 2018.

24 S. Li, J. G. Park, Z. Liang, T. Siegrist, T. Liu, M. Zhang, Q. Cheng, B. Wang and C. Zhang, "In situ characterization of structural changes and the fraction of aligned carbon nanotube networks produced by stretching." Carbon, vol. 50, no. 10, p. 3859-3867, 2012.

25 D. Lellinger, D. Xu, A. Ohneiser, T. Skipa and I. Alig, "nfluence of the injection moulding conditions on the in-line measured electrical conductivity of polymer-carbon nanotube composites." physica status solidi (b), vol. 245, no. 10, p. 2268-2271, 2008.

26 I. Alig, D. Lellinger, M. Engel, T. Skipa and P. Pötschke, "Destruction and formation of a conductive carbon nanotube network in polymer melts: In-line experiments." Polymer, vol. 49, no. 7, p. 1902-1909, 2008.

27 P. C. Ma, N. A. Siddiqui, G. Marom and J. K. Kim, "Dispersion and functionalization of carbon nanotubes for polymer-based nanocomposites: a review." Composites Part A: Applied Science and Manufacturing, vol. 41, no. 10, p. 1345-1367, 2010.

28 J. Obrzut, J. F. Douglas, S. B. Kharchenko and K. B. Migler, "Shear-induced conductor-insulator transition in melt-mixed polypropylene-carbon nanotube dispersions." Physical Review B, vol. 76, no. 19, p. 195420/1-9, 2007.

29 W. Bauhofer, S. Schulz, A. Eken, T. Skipa, D. Lellinger, I. Alig, E. Tozzi and D. Klingenberg, "Shearcontrolled electrical conductivity of carbon nanotubes networks suspended in low and high molecular weight liquids." Polymer, vol. 51, no. 22, p. 5024-5027, 2010.

30 A. Combessis, Charvin, A. N., F. J. A. and L. Flandin, "Understanding dynamic percolation mechanisms in carbonaceous polymer nanocomposites through impedance spectroscopy: Experiments and modeling." Journal of Applied Physics, vol. 116, p. 034103-1-10, 2014.

31 T. Skipa, D. Lellinger, W. Böhm, M. Saphiannikova and I. Alig, "Influence of shear deformation on carbon nanotube networks in polycarbonate melts: Interplay between build-up and destruction of agglomerates." Polymer, vol. 51, no. 1, p. 201-210, 2010.

32 M. Marcourt, P. Cassagnau, R. Fulchiron, D. Rousseaux, O. Lhost and S. Karam, "A model for the electrical conductivity variation of molten polymer filled with carbon nanotubes under extensional deformation." Composites Science and Technology, vol. 168, p. 111-117, 2018. 